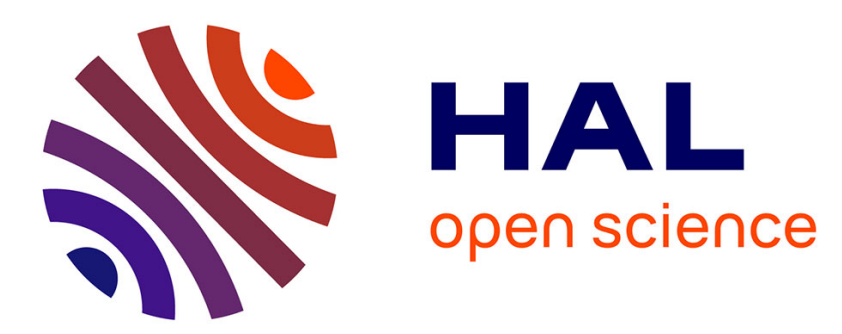

\title{
Les Dégâts alimentaires du chevreuil en milieu forestier.
} Charles Maizeret, Hélène Tixier, Philippe Ballon, Patrick Duncan, Benoît Guibert

\section{To cite this version:}

Charles Maizeret, Hélène Tixier, Philippe Ballon, Patrick Duncan, Benoît Guibert. Les Dégâts alimentaires du chevreuil en milieu forestier.. Revue forestière française, 1999, 51 (3), pp.405-413. $10.4267 / 2042 / 5446$. hal-03443522

\section{HAL Id: hal-03443522 \\ https://hal.science/hal-03443522}

Submitted on 23 Nov 2021

HAL is a multi-disciplinary open access archive for the deposit and dissemination of scientific research documents, whether they are published or not. The documents may come from teaching and research institutions in France or abroad, or from public or private research centers.
L'archive ouverte pluridisciplinaire HAL, est destinée au dépôt et à la diffusion de documents scientifiques de niveau recherche, publiés ou non, émanant des établissements d'enseignement et de recherche français ou étrangers, des laboratoires publics ou privés. 


\title{
LES DÉGÂTS ALIMENTAIRES DU CHEVREUIL EN MILIEU FORESTIER
}

\author{
C. MAIZERET - H. TIXIER - P. BALLON \\ P. DUNCAN - B. GUIBERT
}

L'augmentation constante des cheptels de cervidés depuis une trentaine d'années suscite de vives polémiques entre les forestiers et les chasseurs. Les premiers ont en effet constaté un accroissement important des dégâts sur les régénérations et certains d'entre eux considèrent qu'il leur devient difficile de réaliser des reboisements dans des conditions satisfaisantes (Monomakhoff, 1992 ; Ministère de l'Agriculture, 1994). Le Chevreuil est de plus en plus la cible principale de ces critiques alors que cette espèce était autrefois considérée comme moins dévastatrice que le Cerf. Ce paradoxe peut s'expliquer de deux façons:

- son aire de répartition s'est considérablement étendue et ses densités n'ont cessé d'augmenter depuis plusieurs dizaines d'années (ONC, 1994);

- il se caractérise par une forte sélectivité alimentaire et il recherche particulièrement certaines essences (Maizeret, 1988 ; Tixier et al., 1997).

Ce dernier caractère a certainement joué un rôle important dans l'évolution de la façon dont le Chevreuil est perçu. On comprend en effet la colère du sylviculteur qui constate que tous ses plants de chênes rouges ont été abroutis alors que le reste de la végétation de la parcelle ne semble pas avoir intéressé les animaux.

Les forestiers ont constaté depuis longtemps qu'il suffit qu'une essence nouvelle soit implantée sur leur territoire pour qu'elle soit aussitôt attaquée. II n'en fallait pas plus pour que le Chevreuil soit taxé d'une intentionnalité maligne et que les forestiers s'estiment confrontés à un ennemi sournois qui leur en voulait personnellement...

Des travaux récents, et en particulier ceux qui ont été réalisés au Centre d'Études biologiques de Chizé, ont permis cependant de mieux connaître le comportement alimentaire du Chevreuil (Duncan et al., 1998 ; Cederlund et al., 1998). La compréhension des mécanismes comportementaux qui déterminent ses préférences permet de considérer le problème des dégâts forestiers de façon plus pragmatique.

\section{L'ÉTABLISSEMENT DES PRÉFÉRENCES ET DES AVERSIONS ALIMENTAIRES CHEZ LE CHEVREUIL}

Les processus qui déterminent les choix alimentaires chez le Chevreuil sont de deux types:

- les mécanismes perceptifs, qui ont trait aux sensations produites par les différents stimuli induits par la plante; 


\section{MAIZERET et al.}

- les mécanismes d'apprentissage individuel qui permettent l'élaboration des comportements d'ingestion ou de rejet de chaque catégorie d'aliment.

\section{Les mécanismes perceptifs}

Les caractéristiques physico-chimiques de la plante induisent des stimuli qui concernent quatre des cinq sens de l'animal : la vue, l'odorat, le goût et le toucher.

Les messages sensoriels vont en particulier dans deux directions : I'hypothalamus et le cortex cérébral. Le système limbique de l'hypothalamus est parfois appelé "centre du plaisir". C'est là que se définissent les sensations agréables ou désagréables. Le cortex cérébral intègre quant à lui les informations qui permettent l'identification de la plante et celles qui ont trait aux sensations résultant de son ingestion. On pourrait donc considérer schématiquement que, sur la figure 1 (ci-dessous), le cadre relatif à la tonalité hédonique (formation de la sensation agréable) correspond à l'hypothalamus et que la représentation des aliments correspond au cortex cérébral.

Figure 1 MÉCANISMES IMPLIQUÉS DANS LA PERCEPTION DES STIMULI D'ORIGINE ALIMENTAIRE ET L'IDENTIFICATION DES ALIMENTS

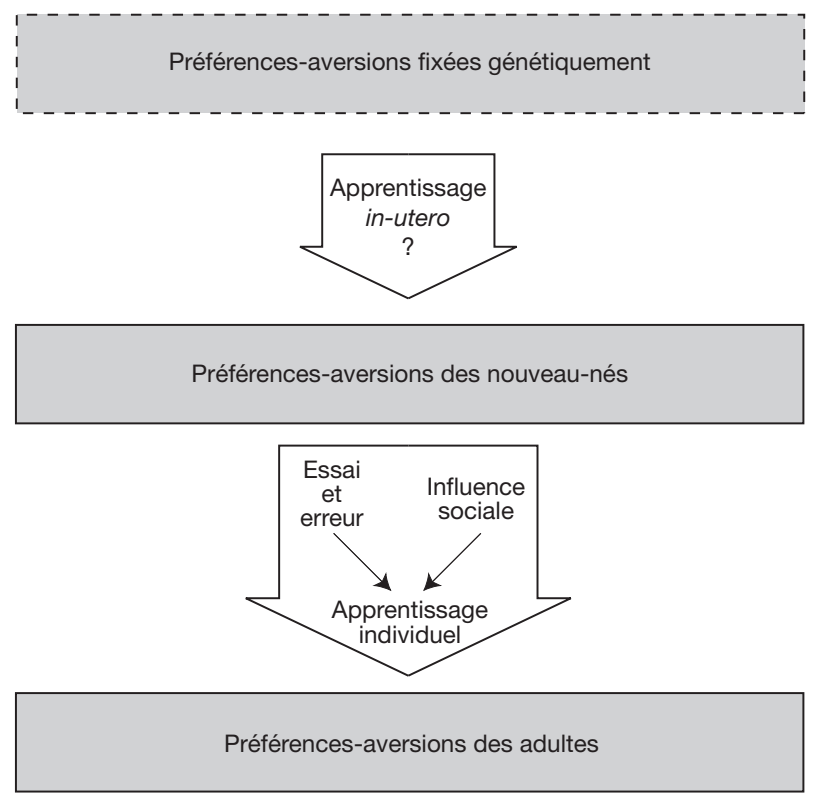

Les stimuli induits par les végétaux consommés sont de trois ordres (figure 1) :

- ceux qui sont perçus lorsque l'animal est en présence de la plante : il s'agit des stimuli visuels et olfactifs. Les connaissances actuelles permettent de penser qu'ils n'induisent pas directement de sensation agréable ou désagréable (Maizeret, 1993). Ainsi, l'odeur, contrairement à une idée répandue, ne permet pas à elle seule d'induire des réactions d'ingestion ou de rejet. Les expériences menées sur des chevreuils en captivité montrent en effet que ceux-ci ne manifestent aucune aversion olfactive vis-à-vis des aliments non consommés. Ce n'est 
qu'après les avoir goûtés que les jeunes faons apprennent à les éviter (Tixier, 1996 ; Tixier et al., 1998). L'odeur constitue par contre un critère de reconnaissance de la plante chez les animaux et elle contribue ainsi à constituer la "carte d'identité" de l'aliment.

- ceux qui sont perçus lors de la prise en bouche de l'aliment : les chémorécepteurs des lèvres et de la cavité buccale renseignent l'animal sur la structure physique de la plante (présence d'épines ou de spicules, rugosité, consistance, résistance à l'écrasement,...) ainsi que sur certaines de ses propriétés chimiques (astringence, caractère irritant,...). Les récepteurs du goût, situés sur la langue, perçoivent les quatre saveurs fondamentales : salé, sucré, acide, amer. Enfin, la voie rétro-nasale, qui met en communication la bouche avec le système olfactif, permet aux molécules volatiles d'atteindre les récepteurs de l'odorat. Tant que les concentrations n'excèdent pas certains seuils, les saveurs sucrées et salées peuvent induire des appétits spécifiques chez les ruminants (Bell, 1959 ; Goatcher et Church, 1970 ; Arnold et Hill, 1972 ; Church, 1979).

Inversement, l'aversion pour l'amertume ou pour l'excès d'acidité est considérée comme commune à tous les vertébrés (Crawford et Church, 1971; Bate-Smith, 1972 ; Garcia et Hankins, 1974).

Figure 2 MÉCANISMES COMPORTEMENTAUX IMPLIQUÉS DANS L'APPARITION DES PRÉFÉRENCES-AVERSIONS (P/A)

PLANTE

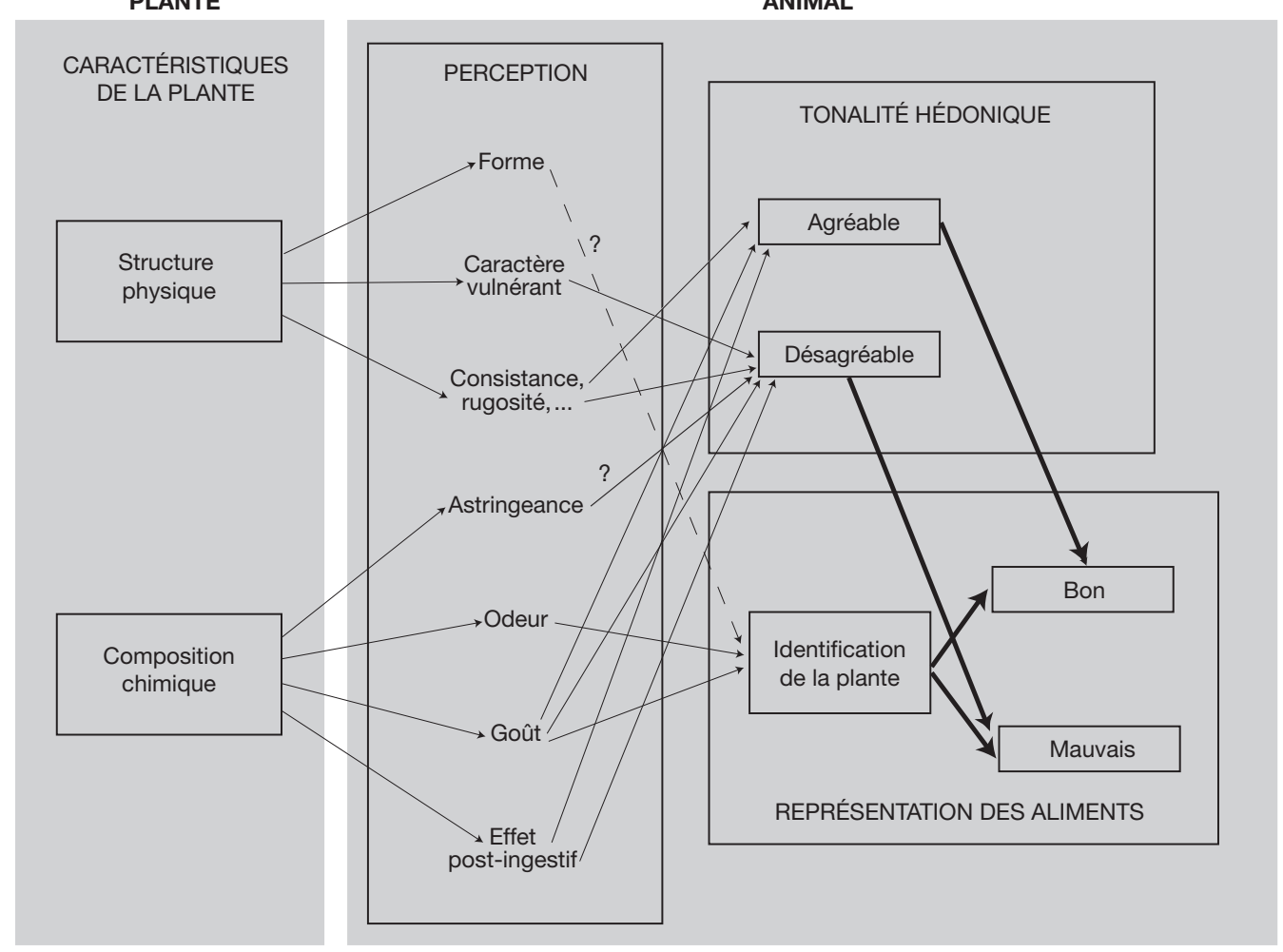




\section{MAIZERET et al.}

Lorsqu'on a présenté différentes espèces végétales à des faons nouveau-nés (Tixier, 1996), on a pu constater que toutes étaient goûtées au moins une fois mais certaines d'entre elles, telles que l'Arum maculé ou l'Euphorbe des bois, ont été rejetées dès les premières prises et n'ont pratiquement plus été consommées par la suite. Cette aversion spontanée a été attribuée à la présence de composés irritants qui constituent des mécanismes très efficaces de défense des plantes vis-à-vis des grands herbivores.

- ceux qui sont perçus une fois que l'aliment est avalé : il se produit alors un effet post-ingestif qui se traduit par une sensation agréable si l'aliment possède une bonne valeur nutritionnelle (satiété) ou, s'il est toxique, par une sensation de malaise (douleur intestinale, effet neurotoxique,...).

Les mécanismes d'apprentissage individuel (figure 2, p. 407)

On a considéré pendant très longtemps que les ongulés sauvages disposaient d'une "sagesse nutritionnelle" innée (Albrecht, 1945 ; Stapleton, 1947). Les résultats des études récentes semblent indiquer que la composante préétablie du comportement alimentaire est assez réduite et que c'est surtout par un mécanisme d'apprentissage individuel que celui-ci se développe (Maizeret, 1993).

Les capacités de discrimination des tout jeunes animaux paraissent essentiellement limitées à l'évitement des plantes vulnérantes ou irritantes ainsi que de celles qui ont une saveur désagréable (amertume ou acidité fortes). II est cependant possible qu'un apprentissage très précoce puisse déterminer certaines préférences chez le Chevreuil. II a en effet été constaté chez d'autres espèces de mammifères que des composés issus de l'alimentation de la mère pouvaient être transmis par le lait et influencer ainsi durablement les préférences du jeune (Galef et Sherry, 1973 ; Galef, 1976).

À Chizé, il a été constaté que les choix alimentaires des nouveau-nés "naïfs" sont différents de ceux des adultes. Ce n'est que dans le courant du mois qui suit la mise en présence de la végétation que les préférences se rapprochent progressivement de celles des adultes (figure 3 , p. 409). Cette évolution a été attribuée à un processus d'apprentissage par essai et erreur: l'animal n'ingère tout d'abord que de petites quantités d'un aliment nouveau et il attend de sentir les effets produits. Si l'aliment est toxique, il provoque une sensation de malaise et il est évité à l'avenir. Si, par contre, l'effet post-ingestif est positif, l'aliment pourra être consommé en quantités croissantes.

Ce mécanisme joue probablement un rôle prépondérant dans l'établissement des préférences alimentaires et il permet à celles-ci d'être parfaitement adaptées aux besoins nutritionnels de l'animal.

\section{LE DÉTERMINISME DES DÉGÂTS D'ABROUTISSEMENT}

\section{Les dégâts du point de vue du forestier et du point de vue du Chevreuil}

Par hypothèse, considérons le cas d'une plantation de Chênes pédonculés à une densité de 1100 plants/ha, dont toutes les pousses terminales auraient été abrouties. Au cours des tests d'appétence des plants feuillus qui ont été réalisés à Chizé (Ballon et al., 1999), il a été mis en évidence que la consommation d'une pousse de Chêne pédonculé en pleine période de végétation correspond à un prélèvement moyen de 2,0 $\mathrm{g}$ de matière sèche. Partant de ce constat, l'abroutissement de tous les plants d'une régénération d'un hectare ne procure au chevreuil que 2,2 kg de matière sèche, ce qui correspond en fait aux besoins nutritionnels d'un animal pendant cinq jours environ (Boisaubert et Boutin, 1988). 


\section{ANALYSE DES CORRESPONDANCES MONTRANT L'ÉVOLUTION \\ DE LA CONSOMMATION DE SEPT ESPĖCES DE PLANTES PAR NEUF FAONS au cours de douze tests réalisés de l'âge de deux à six semaines \\ Lorsque les faons sont très jeunes ( $1^{\text {er }}$ et $2^{\mathrm{e}}$ tests), ils ont des régimes différents de ceux des animaux adultes (zone hachurée). \\ Par la suite, leurs choix se rapprochent de ceux des adultes.}

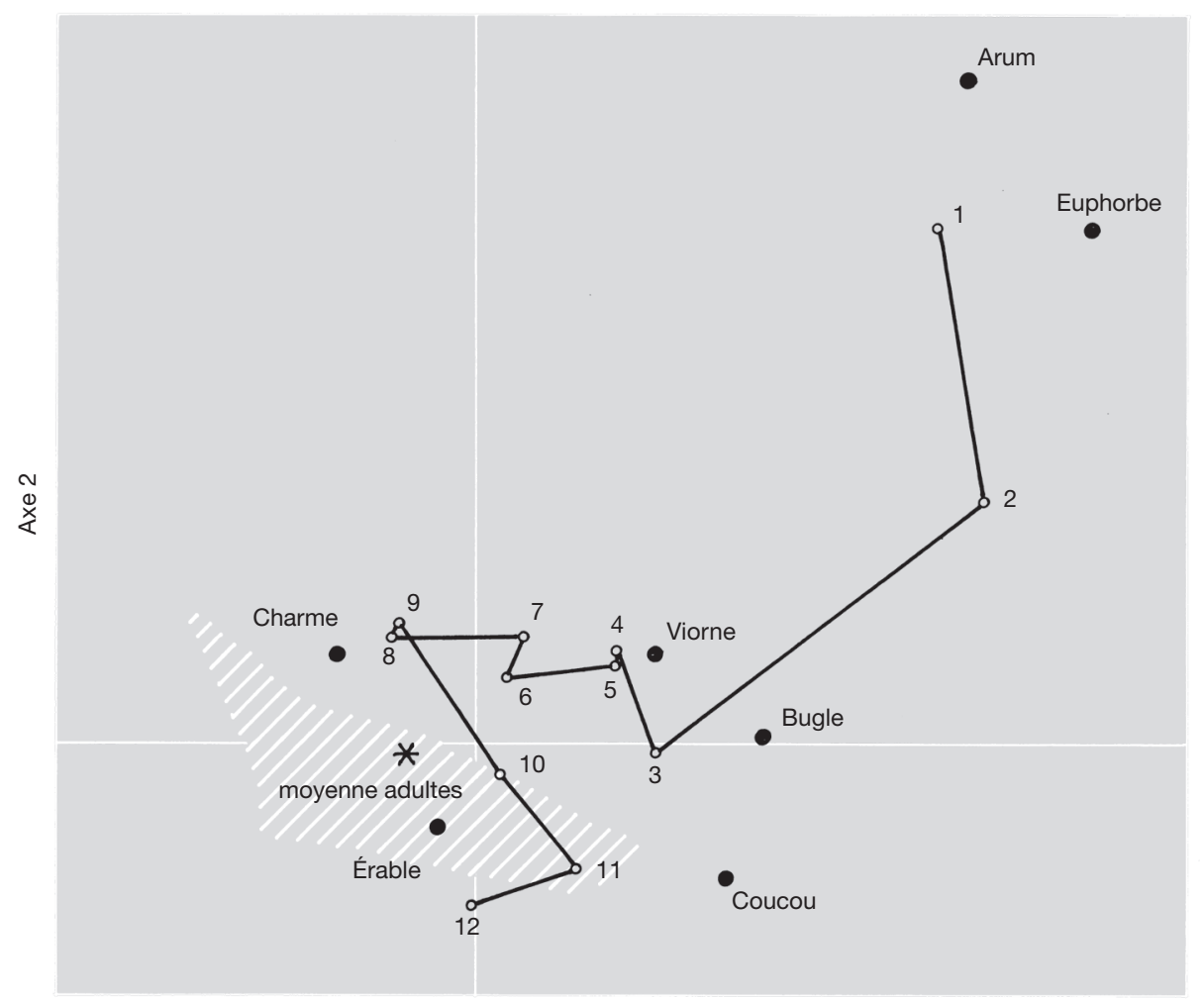

Axe 1

Cet exemple montre que, en matière de dégâts, il est essentiel de relativiser l'apport nutritionnel que constitue la consommation des plants forestiers. Pour le propriétaire de la parcelle, les dommages sont importants puisque la totalité des plants risquent d'être déformés. Le Chevreuil, lui, n'a fait que grignoter...

\section{Les causes de la forte appétence de certains plants forestiers}

Même s'il n'en a retiré qu'une petite partie de sa ration alimentaire, le Chevreuil de l'exemple précédent a malgré tout consommé méthodiquement toutes les pousses de chênes les unes après les autres. II a donc fait preuve d'un comportement de recherche active des plants et on peut se demander pourquoi ceux-ci attirent à ce point les animaux.

L'analyse comparative de la valeur nutritionnelle des semis naturels et des plants issus de pépinières (Ballon et Boscardin, 1995) a montré que le Chevreuil a intérêt à préférer les derniers, surtout s'ils ont bénéficié des techniques les plus modernes d'élevage. Ils ont eu en effet une croissance accélérée, leurs pousses sont donc peu fibreuses et elles sont riches en sucres ainsi qu'en sels minéraux. 


\section{MAIZERET et al.}

\section{L'attrait des essences nouvelles}

Avant d'affirmer que les chevreuils d'un territoire ont une attirance marquée pour une essence nouvellement introduite, il faut tout d'abord vérifier le phénomène par des relevés précis. Si les tests confirment que les animaux ingèrent systématiquement et en quantités importantes des aliments qui sont nouveaux pour eux, il y a de fortes chances pour que cette situation soit le signe d'un problème alimentaire.

En effet, le Chevreuil manifeste habituellement un comportement de prudence vis-à-vis des aliments qu'il ne connaît pas (Müri, 1978). Cette réaction lui évite d'ingérer de trop grandes quantités de composés secondaires toxiques. Ce n'est qu'après avoir expérimenté leur innocuité qu'il intègre les nouveaux aliments dans son régime. Le fait de supprimer cette phase d'expérimentation comporte donc un risque important pour les chevreuils. Ce risque n'est probablement pris que sous la contrainte d'une situation alimentaire difficile. Celle-ci peut avoir deux origines différentes:

- soit le milieu est naturellement très pauvre et les animaux ne parviennent pas à subvenir à leurs besoins nutritionnels à certaines périodes de l'année ;

- soit la densité est trop importante et la végétation disponible est surexploitée.

\section{CONCLUSION : PERSPECTIVES POUR UNE MEILLEURE COHABITATION ENTRE LES CHEVREUILS ET LES FORESTIERS}

Proportionnellement à sa taille, le Chevreuil a des besoins nutritionnels importants. Pour satisfaire à ces exigences, il a développé un comportement alimentaire très élaboré qui lui permet de sélectionner les aliments les plus profitables. Lorsqu'on introduit à l'intérieur de son territoire des plants forestiers artificiellement enrichis, il ne faut donc pas s'étonner que ceux-ci fassent l'objet d'une consommation préférentielle. Dans de pareils cas, les mesures de réduction des effectifs sont souvent peu efficaces car un seul animal peut très bien ravager une plantation à lui tout seul. La seule solution est donc d'utiliser des protections individuelles.

Le cas des dégâts sur les autres types de régénérations (semis naturels) ou sur les recépages est différent: l'importance des attaques dépend souvent directement de la densité des animaux et le prix des protections est prohibitif. Dans une perspective de développement durable, il semble difficile de laisser une seule espèce porter atteinte à la conduite sylvicole normale d'un peuplement. Le rôle du plan de chasse est précisément de maintenir les effectifs au niveau économiquement supportable. Du point de vue cynégétique, de nombreuses études ont par ailleurs montré que les situations de surdensité entraînent une baisse des disponibilités alimentaires qui se traduit à son tour par une réduction du poids des animaux, de la productivité, du taux de survie des jeunes, etc. (Maizeret et al., 1989 ; Vincent et al., 1995). Personne ne semble donc avoir intérêt à maintenir des densités trop fortes.

Il est cependant très difficile de savoir, sur un territoire donné, si les animaux sont réellement plus nombreux que ce qu'il ne faudrait. Les méthodes de dénombrement sont en effet très lourdes à mettre en œuvre et les connaissances sur les relations entre les animaux et la végétation sont de toutes façons insuffisantes pour pouvoir apprécier à partir de quelle densité les effets néfastes peuvent apparaître.

Pour pallier ces difficultés, les biologistes orientent de plus en plus leurs recherches vers la mise au point de méthodes indiciaires qui rendent compte de l'état d'équilibre forêt-gibier (Groupe chevreuil, 1996). Le principe est simple : on ne cherche plus à savoir combien il y a 
d'animaux, ni même combien il en faudrait, mais on s'efforce simplement de discerner si la population du territoire considéré n'est pas trop importante par rapport à ce que le milieu peut supporter.

La plupart des méthodes indiciaires qui ont été développées jusqu'à présent l'ont été à l'initiative des organismes cynégétiques et elles se basent sur des indices qui concernent essentiellement la biologie des animaux (disponibilités alimentaires, caractéristiques biométriques des différentes classes d'âges, nombre de jeunes par femelle, taille des groupes,...). Les forestiers auraient intérêt à mettre au point de la même façon des méthodes qui seraient basées sur des indices à caractère sylvicole qui rendraient compte de l'influence des animaux sur la conduite "normale" des peuplements (densité de semis intacts par rapport au recrutement nécessaire, taux de déformation ou de dépérissement des tiges, etc.).

S'il s'avère possible de mettre au point des techniques de relevés de terrain qui soient suffisamment simples à mettre en œuvre, les méthodes indiciaires devraient contribuer efficacement à normaliser les relations entre les chasseurs et les sylviculteurs. En effet, les observations pourraient alors être réalisées conjointement par le personnel technique des deux catégories d'organismes, ce qui enlèverait toute possibilité de discussion sur la réalité des problèmes constatés.

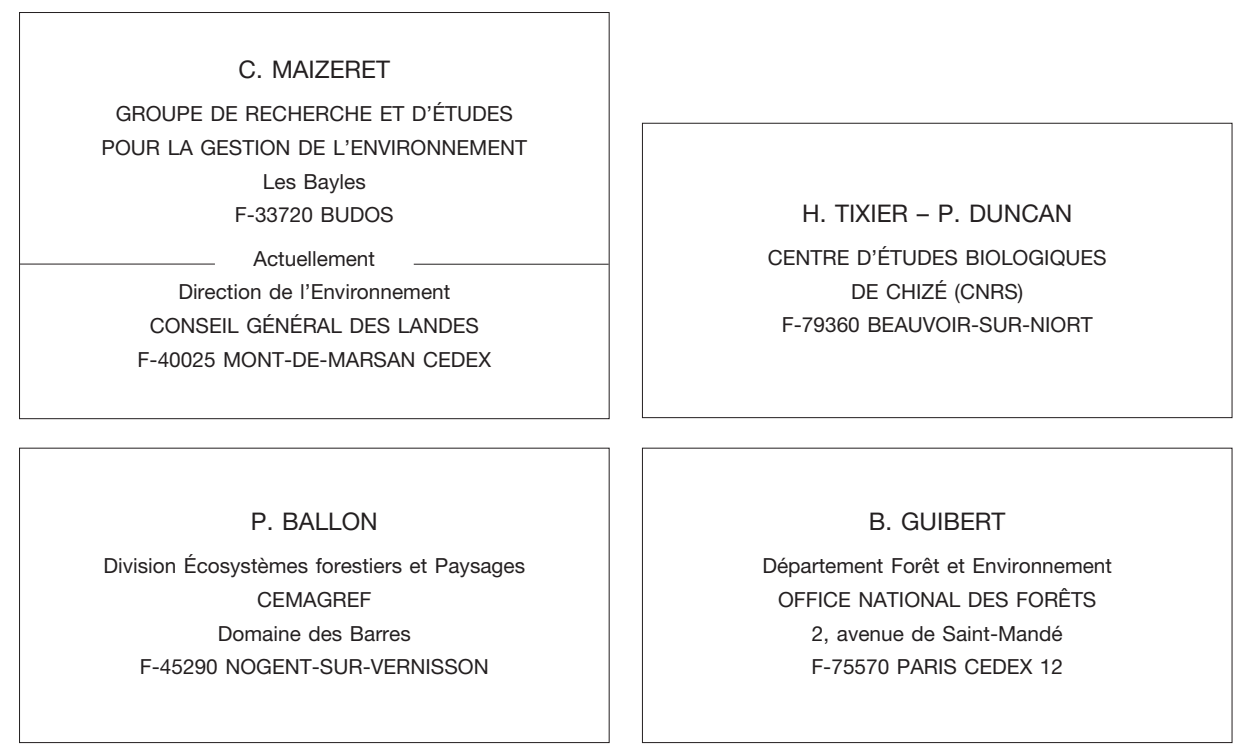

\section{BIBLIOGRAPHIE}

ALBRECHT (W.A.). - Discrimination on food selection by animals. - Sci. Monthly, vol. 60, 1945, pp. $347-352$. ARNOLD (G.W.), HILL (J.L.). - Chemical factors affecting selection of food plants by ruminants. In : Phytochemical Ecology / J.B. Harbone Ed. - New-York: Academic Press, 1972. - pp. 71-101.

BALLON (P.), BOSCARDIN (Y.). - Rôle de la composition chimique des plants forestiers sur leur niveau de consommation par le chevreuil. In : XXII' Congrès de l'IUGB, Sofia, Bulgarie, septembre 1995, pp. 203-209. 


\section{MAIZERET et al.}

BALLON (P.), GUIBERT (B.), AMARD (J.-P.), GUILLON (N.), GUILLON (N.), BOSCARDIN (Y.). - Sensibilité de quelques essences forestières de reboisement à l'abroutissement par le Chevreuil (Capreolus capreolus). Revue forestière française, vol. LI, $\mathrm{n}^{\circ} 1,1999$, pp. 20-34.

BATE-SMITH (E.C.). - Attractants and repellents in higher animals. In : Phytochemical Ecology / J.B. Harbone Ed. - New-York: Academic Press, 1972. - pp. 45-57.

BELL (F.R.). - Preference thresholds for taste discrimination in goats. - Journal of agricultural Science, 52, 1959, pp. 125-129.

BOISAUBERT (B.), BOUTIN (J.-M.). - Le Chevreuil. - Paris : Hatier, 1988. - 236 p.

CEDERLUND (G.), BERGSTRÖM (R.), KJELLANDER (P.), GILL (R.M.A.), GAILLARD (J.-M.), BOISAUBERT (B.), BALLON (P.), DUNCAN (P.). - Managing roe deer and their impact on the environment. In: The European roe deer : the biology of success / R. Andersen, P. Duncan, J.D.C. Linnel Eds. - Scandinavian University Press, 1998.

$\mathrm{CHURCH}$ (D.C.). - Taste, appetite, regulation of energy balance and control of food intake. In : Digestive physiology and nutrition of ruminants, vol. 2.0 / B. Books Eds. - Corvallis, 1979. - pp. 281-290.

CRAWFORD (J.C.), CHURCH (D.C.). - Response of black-tailed deer to various chemical taste stimuli. J. Wildlife Management, vol. 35, $\mathrm{n}^{\circ}$ 2, 1971, pp. 210-215.

DUNCAN (P.), TIXIER (H.), HOFFMAN (R.R.), LECHNER-DOLL (M.). - Feeding strategies and the physiology of digestion in roe deer. In: The European roe deer: the biology of success / R. Andersen, P. Duncan, J.D.C. Linnel Eds. - Scandinavian University Press, 1998.

GALEF (B.G.). - The social transmission of acquired behavior : a discussion of tradition and social learning in vertebrates. - Advances in the Study of behavior, vol. 6, 1976, pp. 77-100.

GALEF (B.G.), SHERRY (D.F.). - Mother's milk : a medium for transmission of cues reflecting the flavor of the mother's diet. - Journal of comparative and physiological Psychology, vol. 83, 1973, pp. 374-378.

GARCIA (J.), HANKINS (W.G.). - The evolution of bitter and the acquisition of toxiphobia. In : Fifth International Symposium on olfaction and taste / D.A. Denton, J.P. Coghlan Eds. - New-York : Academic Press, 1974. pp. 39-45.

GOATCHER (W.D.), CHURCH (D.C.). - Review of some nutritional aspect of the sense of taste. - J. Animal Sci., vol. 31, 1970, pp. 973-981.

GROUPE CHEVREUIL. - Les Bio-indicateurs : futurs outils de gestion des populations de chevreuils ? - Bulletin mensuel de l'Office national de la Chasse, supplément au n²09, fiche technique $n^{\circ}$ 90, 1996, 2 p.

MAIZERET (C.). - L'Aversion alimentaire chez les mammifères : ontogénèse et utilisation pour la prévention des dégâts de cervidés. - Gibier Faune sauvage, vol. 10, 1993, pp. 217-227.

MAIZERET (C.). - Stratégies alimentaires des chevreuils : les fondements écologiques d'une diversification du régime. - Acta Oecologica. Oecologia applicata, n 9, 1988, pp. 191-211.

MAIZERET (C.), BOUTIN (J.-M.), CIBIEN (C.), CARLINO (J.P.). - Effects of population density on the diet of roe deers and the availability of their food in Chizé forest. - Acta theriologica, vol. 34, $\mathrm{n}^{\circ} 16,1989, \mathrm{pp} .235-246$.

MINISTÈRE DE L'AGRICULTURE (DERF). - La Gestion durable des forêts françaises. - Paris : Ministère de l'Agriculture - Direction de l'Espace rural et de la Forêt, 1994. - 76 p.

MONOMAKHOFF (P.). - Les Dégâts de cervidés en forêt, ce qu'ils nous coûtent. - Communes forestières de France, $\mathrm{n}^{\circ}$ 1, 1992, pp. 3-13.

MÜRI (H.). - Observations and experiments on learning process in food choice of roe-deer. $-Z$. Sauegetierkd, vol. $43, n^{\circ} 3,1978$, pp. 171-186.

ONC. - Tableaux de chasse cerfs, chevreuils, sangliers, saison 1993-1994. - Supplément au Bulletin mensuel de l'Office national de la Chasse, $\mathrm{n}^{\circ} 194,1994,4 \mathrm{p}$.

STAPLETON (R.G.). - The palatibility and nutritive value of herbage plants. - J. Mineral. Agric., vol. 53, 1947, pp. 425-431.

TIXIER (H.). - Déterminants et ontogénèse du comportement alimentaire du Chevreuil (Capreolus capreolus). Université de Paris Nord, 1996. - 211 p. (Thèse).

TIXIER (H.), DUNCAN (P.), GUILLON (N.), GUILLON (N.). - Sélection alimentaire chez le Chevreuil, influence des caractéristiques physiques et chimiques de la végétation. - Bulletin mensuel de l'Office national de la Chasse, $\mathrm{n}^{\circ}$ 220, 1997, pp. 10-21.

TIXIER (H.), DUNCAN (P.), SCEHOVIC (J.), YANI (A.), GLEIZES (M.), LILA (M.). - Food selection by European roe deer : effects of plants chemistry and consequences for the nutritional value of their diets. $-\mathrm{J}$. Zool., $\mathrm{n}^{\circ} 242$, 1997, pp. 229-245.

TIXIER (H.), MAIZERET (C.), DUNCAN (P.), BERTRAND (R.), POIREL (C.), ROGER (M.). - Development of feeding selectivity in roe deer. - Behavioural Processes, $n^{\circ} 43,1998$, pp. 33-42.

VINCENT (J.-P.), BIDEAU (E.), HEWISON (A.J.M.), ANGIBAULT (J.M.). - The influence of increasing density on body weiht, kid production, home range and winter grouping in roe deer. - J. Zool., n² 236, 1995, pp. 371-382. 


\section{LES DÉGÂTS ALIMENTAIRES DU CHEVREUIL EN MILIEU FORESTIER (Résumé)}

Diverses études qui ont été conduites ces dernières années apportent un éclairage nouveau au problème des dégâts forestiers causés par le Chevreuil. Dans le cas des plantations, il a pu être montré que l'abroutissement est déterminé par la qualité nutritionnelle des pousses mais l'apport alimentaire de celles-ci reste malgré tout très faible en regard des besoins quotidiens des animaux. Au moins pour les essences les plus appétentes, il semble donc illusoire d'espérer supprimer les dégâts par une simple augmentation des plans de chasse. La seule solution est d'utiliser des protections individuelles.

Dans le cas des dégâts sur les semis ou les recépages, par contre, l'adaptation des effectifs s'impose pour permettre une régénération normale des peuplements.

\section{DAMAGE DUE TO FEEDING BY ROE DEER IN FOREST ENVIRONMENTS (Abstract)}

Various surveys conducted in recent years are shedding new light on the problem of damage to forests caused by roe deer. In the case of plantations, it has been shown that browsing is determined by the nutritive quality of the shoots, but their contribution to the daily needs of these animals is in any case slight. For the most appetible species, it therefore seems unlikely that increasing hunting entitlements will in itself solve the problem. The only solution is individual protection.

However, in the case of damage on seedlings and coppices, regulating the deer population is needed for normal regeneration of stands to take place.

\section{- ERRATUM}

Dans la bibliographie de l'article de Ph. BALLON, B. GUIBERT, J.-P. HAMARD, N. GUILLON, Nadine GUILLON, Y. BOSCARDIN : "Sensibilité de quelques essences forestières de reboisement à l'abroutissement par le Chevreuil (Capreolus capreolus)", paru dans le numéro $1 / 1999$ de la Revue forestière française, il faut citer la référence Ballon, Klein, Saint-Andrieux comme suit :

KLEIN (F.), SAINT-ANDRIEUX (Ch.), BALLON (Ph.). - Quelles protections pour les plants forestiers? - Bulletin mensuel de l'ONC, n 141, décembre 1989, pp. 31-35. 